\title{
A Measure of Arbitrariness in Abductive Explanations
}

\author{
Luciano Caroprese, Irina Trubitsyna \\ DIMES, Università della Calabria, Cosenza, Italy \\ (e-mail: caroprese, irina@deis.unical.it) \\ Mirosław Truszczyński \\ Department of Computer Science, University of Kentucky, Lexington, USA \\ (e-mail: mirek@cs.uky.edu) \\ Ester Zumpano \\ DIMES, Università della Calabria, Cosenza, Italy \\ (e-mail: zumpano@deis.unical.it)
}

submitted 1 January 2003; revised 1 January 2003; accepted 1 January 2003

\begin{abstract}
We study the framework of abductive logic programming extended with integrity constraints. For this framework, we introduce a new measure of the simplicity of an explanation based on its degree of arbitrariness: the more arbitrary the explanation, the less appealing it is, with explanations having no arbitrariness - they are called constrained - being the preferred ones. In the paper, we study basic properties of constrained explanations. For the case when programs in abductive theories are stratified we establish results providing a detailed picture of the complexity of the problem to decide whether constrained explanations exist.

To appear in Theory and Practice of Logic Programming (TPLP).
\end{abstract}

\section{Introduction}

Abduction is an important form of nonmonotonic reasoning. The concept was introduced in the late 19th century by the American philosopher Charles Sander Peirce (1995) as an inference scheme aimed at deriving potential explanations of observations 1 A general characteristic of abductive reasoning is the existence of multiple abductive explanations, which are typically not equally compelling. Therefore, identifying a subclass, possibly narrow, of "preferred explanations" is an important problem. Following the Occam's principle, a typical approach is to identify as "preferred" those explanations that are, in some sense, simple. Several concepts of simplicity were considered in the literature, most notably those based on minimality with respect to inclusion and cardinality. In the context of logic programming, abduction was first studied by Eshghi and Kowalski (1989), and then by Kakas and Mancarella (1990b) under the brave reasoning variant of the stable-model semantics. That work established abductive logic programming as an important subarea of abduction, where the background theory is represented by a logic program, often with negation in the bodies and disjunction in the heads, under any of the standard logic programming semantics (Kakas et al. 1992; Denecker and Kakas 2002). This paper is concerned with the problem of limiting the space of explanations in the framework of abductive

\footnotetext{
${ }^{1}$ Peirce gave abduction the following informal interpretation: "The surprising fact, $C$, is observed; but if $A$ were true, $C$ would be a matter of course: hence, there is reason to suspect that $A$ is true."
} 
logic programming extended by integrity constraints. We introduce a new measure of the quality of an explanation in terms of its arbitrariness and propose to consider as "preferred" only those explanations that minimize arbitrariness. Our approach can be applied with any of the standard semantics of logic programs.

\section{Example 1}

Let us consider the following scenario. It is Saturday and Bob is known to ski on Saturdays if it is not snowing. And when he is not skiing, he is on campus. We can represent this information by the logic program:

$$
\text { \{ saturday. } \quad \text { skiing } \leftarrow \text { saturday, } \text { not } \text { snows. } \quad \text { on_campus } \leftarrow \text { not skiing. }\}
$$

If it's Saturday and we see Bob on campus, we can abduce it is snowing. Otherwise, Bob would be skiing. To put it differently, given our background knowledge, the fact snows is an abductive explanation (explanations will be formally defined in the next section) to our observation on_campus.

In this example, there is only one explanation of the observation (assuming all that is relevant to our reasoning has been mentioned). However, a general feature of abductive reasoning is the existence of multiple explanations. Typically, they are not all equally likely. Thus, narrowing down the range of possible explanations to a smaller set of "most likely" or "preferred" ones becomes an important problem. The key to it is a well-motivated notion of "preferred". We will now present an example meant to develop intuitions behind the notion of "preferredness".

\section{Example 2}

Let us consider the following scenario modeling security breaches in an information system. A security breach at a component of the system may only occur when a person with an account makes an unauthorized access. Regular staff personnel have accounts on the system if they complete training and have their security clearance current. Visitors may also be granted an account but only with an approval by the head of the IT department. This situation can be described by the following program:

$$
\begin{aligned}
\operatorname{account}(X) & \leftarrow \operatorname{staff}(X), \operatorname{trained}(X), \operatorname{current}(X) . \\
\operatorname{account}(X) & \leftarrow \operatorname{visitor}(X), \operatorname{approved}(X) . \\
\operatorname{security} \_ \text {breach }(W) & \leftarrow \text { unauthorized_access }(W, X), \operatorname{account}(X) .
\end{aligned}
$$

Let us also assume that tom and mary are regular staff members and dan is a visitor (there may also be other individuals in these groups and additional ones not mentioned as staff or visitors in the program), and that the system has information that tom completed training. That is, the program also contains facts

$$
\text { staff(tom). staff (mary). visitor(dan). trained(tom). }
$$

If we observe security_breach(warehouse) (the security of warehouse was compromised), there are several possible explanations. Below we list some of them:

$$
\begin{aligned}
E_{\text {tom }} & =\{\text { unauthorized_access }(\text { warehouse }, \text { tom }), \text { current }(\text { tom })\} \\
E_{\text {mary }} & =\{\text { unauthorized_access }(\text { warehouse }, \text { mary }), \text { trained }(\text { mary }), \text { current }(\text { mary })\} \\
S_{u} & =\{\text { unauthorized_access }(\text { warehouse }, U), \operatorname{staff}(U), \text { trained }(U), \text { current }(U)\},
\end{aligned}
$$
where $U$ is a name in the domain, possibly not mentioned in the program, 


$$
\begin{aligned}
E_{\text {dan }}= & \{\text { unauthorized_access }(\text { warehouse,dan }), \text { approved }(\text { dan })\} \\
V_{u}= & \{\text { unauthorized_access }(\text { warehouse }, U), \text { visitor }(U), \text { approved }(U)\}, \\
& \text { where } U \text { is a name in the domain, possibly not mentioned in the program } \\
E_{\text {tom,dan }}= & \left\{\begin{array}{l}
\text { unauthorized_access }(\text { warehouse }, \text { tom }), \text { current }(\text { tom }), \\
\text { unauthorized_access }(\text { warehouse }, \text { dan }), \text { approved }(\text { dan })\} .
\end{array}\right.
\end{aligned}
$$

The key question is whether there are principled ways to eliminate some of these explanations as less likely than others.

Most approaches to the problem of selecting preferred explanations follow the Occam's principle of parsimony that entities should not be multiplied unnecessarily and that among possible explanations the simplest one tends to be the right one. The first part of that principle is noncontroversial. However, simplicity is a notoriously complex concept and different formalizations of it are possible! They range from the standard one based on the subset minimality, to its versions and refinements that require minimum cardinality, minimum weight, or minimality under prioritization of individual hypotheses (Eiter et al. 1997). In our example, the explanations $E_{\text {tom }}$, $E_{\text {mary }}, S_{U}, U \neq$ tom, $E_{d a n}$, and $V_{U}, U \neq d a n$, are subset minimal and so, preferred under the subset minimality criterion. The explanations $S_{\text {tom }}, V_{d a n}$, and $E_{\text {tom,dan }}$ are not. If we use a more restrictive criterion of minimum cardinality, the preferred explanations are $E_{\text {tom }}$ and $E_{\text {dan }}$. Let us assume that there are reasons to view each of them as wrong (tom and dan can conclusively demonstrate they were not involved). Under the subset minimality criterion, we now prefer explanations $E_{\text {mary }}, S_{U}, U \neq t o m$, and $V_{U}, U \neq d a n$, while under the minimum cardinality criterion $E_{\text {mary }}$ and $V_{U}, U \neq$ dan, are preferred.

Let us look more carefully at these last two types of explanations. They are specific in that they speak about concrete individuals. However, the explanations of the latter type select an arbitrary individual in the domain (explicitly mentioned or not, as long as it is not dan), with no particular reason to choose one over another. In other words, investigators of the security breach would have no reason to start their investigation with any particular person and the person they would consider first would be arbitrarily selected. On the other hand, the explanation $E_{d a n}$ connects the structural information present in the program and the knowledge provided by the observation in a non-arbitrary (constrained) way - it "invents" no new entities and makes no arbitrary assumptions. Arguably, it would be the explanation pursued first by the investigators.

One might argue that the family of explanations $V_{U}$ simply points to the existence of a person who managed to pass as a visitor with an approval for an account and made an unauthorized access to the IT system, and that this family of explanations can be represented as a single explanation involving existentially quantified formulas (along the lines of an early work by Pople (1973)). However, such "existential”" explanations while no longer making arbitrary choices lack specificity.

In this paper we develop these intuitions into a formal measure of the quality of explanations based on how arbitrary they are. To this end, we introduce the notion of the degree of arbitrariness of an explanation: the smaller that value, the less arbitrary the explanation. We define constrained explanations as those with 0 degrees of arbitrariness, and propose them as preferred. Our most significant technical results establish the computational complexity of the problems to decide whether a given explanation is constrained, and whether a constrained explanation exists. We study the two problems for the case when programs modeling background knowledge in abductive theories are stratified. This is a common case in abductive logic programming. 
From the technical standpoint it has two interesting aspects. First, the two main semantics of logic programs, the stable-model semantics (Gelfond and Lifschitz 1988) and the well-founded semantics (Van Gelder et al. 1991), as well as several others (but not the supported-model semantics (Marek and Subrahmanian 1992)) collapse in this case. Second, when the background knowledge is modeled by a stratified program under the stable-model semantics, all general semantics-independent schemas to define aductive explanations yield the same result. We show that for abductive theories with stratified logic programs, the two computational problems mentioned above are coNP-complete and $\Sigma_{2}^{P}$-complete, respectively. By considering special classes of stratified programs, we establish the main sources of the complexity of the problem.

The remainder of the paper is organized into five sections. First, we recall basic concepts of abductive logic programming and discuss four general schemata to define explanations. Next, we introduce the key concepts for the paper: the degree of arbitrariness in an explanation, and then arbitrary and constrained explanations. The following section presents our main complexity results. Next, we discuss related work where, in particular, we note that our results are also relevant in the area of view update repairs. We conclude by summarizing our contributions and pointing out problems for future work.

\section{Abductive Logic Programs}

We consider a fixed vocabulary $\sigma$ consisting of relation and constant symbols (no function symbols). We write $\mathscr{D}$ for the set of constants in $\sigma$ and assume that $\mathscr{D}$ is an infinite countable set (in some examples, for the sake of simplicity of presentation, we take $\mathscr{D}$ to be finite). We write $\mathscr{H}$ for the set of predicate symbols in $\sigma$. For $\mathscr{Q} \subseteq \mathscr{H}$, we define $\mathscr{Q}^{\mathscr{D}}$ to be the set of all ground atoms whose predicate symbols are in $\mathscr{Q}$. In particular, $\mathscr{H}^{\mathscr{D}}$ is the Herbrand base of $\sigma$.

By $S$ we denote a semantics of logic programs. For now we do not commit to any particular semantics. As usual, we assume only that $S$ is given in terms of Herbrand interpretations of $\sigma$, that is, subsets of $\mathscr{H}^{\mathscr{D}}$. For a logic program $\mathscr{P}$ (in the vocabulary $\sigma$ ), we denote by $\operatorname{sem}_{S}(\mathscr{P})$ the set of Herbrand interpretations of $\sigma$ that are models of $\mathscr{P}$ according to the semantics $S$. Since $\sigma$ is fixed, we omit it from the notation. The most common choice for $S$ is the stable-model semantics (Gelfond and Lifschitz 1988).

We now recall the concept of an abductive theory in the logic programming setting.

\section{Definition 1 (ABDUCTIVE THEORY)}

An abductive theory $\mathscr{T}$ over a vocabulary $\sigma$ is a triple $\langle\mathscr{P}, \mathscr{A}, \mathscr{C}\rangle$, where

- $\mathscr{P}$ is a finite logic program over $\sigma$

- $\mathscr{A} \subseteq \sigma$ is a finite set of predicate names called the abducible predicates

- $\mathscr{C}$ is a finite set of first-order sentences over $\sigma$ called integrity constraints,

and where every rule in $\mathscr{P}$ with an abducible predicate in the head is a ground fact.

Informally, the program $\mathscr{P}$ and the integrity constraints $\mathscr{C}$ provide a model (description) of the problem domain. The program $\mathscr{P}$ defines non-abducible predicates (those not in $\mathscr{A}$ ) in terms of abducible predicates (those in $\mathscr{A}$ ). The integrity constraints in $\mathscr{C}$ impose domain constraints on abducible and non-abducible predicates in the language. According to the definition, information about abducible predicates is given in terms of ground facts. They explicitly specify the extensions of abducible predicates. We refer to ground atoms based on abducible predicates as 
abducibles. An observation is a set of ground facts based on non-abducible predicates. An observation may "agree" with the program $\mathscr{P}$ and the integrity constraints $\mathscr{C}$. But if it does not, we assume that this "disagreement" is caused by the incorrect information about the properties modeled by the abducible predicates. Abductive reasoning consists of inferring updates to the set of abducibles in the program (removal of some and inclusion of some new ones) so that the updated program, the integrity constraints and the observation "agree." Each update that yields an agreement constitutes a possible explanation of the observation. Several notions of agreement have been proposed. They are defined in terms of entailment and satisfiability. A program $\mathscr{P}$ is consistent in the semantics $S$ if $\operatorname{sem}_{S}(\mathscr{P}) \neq \emptyset$. A consistent logic program $\mathscr{P}$ skeptically entails a sentence $\varphi$ (in the same language as $\mathscr{P}$ ), written $\mathscr{P} \models_{S} \varphi$, if for every $M \in \operatorname{sem}_{S}(\mathscr{P}), M \models \varphi$. A consistent logic program $\mathscr{P}$ skeptically entails a set $\Phi$ of sentences, written $\mathscr{P} \models_{S} \Phi$, if $\mathscr{P} \models_{S} \varphi$, for every $\varphi \in \Phi$.

We now present four concepts of "agreement", three of which have received significant attention in the literature.

Definition 2 (AGREEMENT A (DENECKER AND KAKAS 2002))

Let $\mathscr{P}$ be a program and $\mathscr{C}$ a set of integrity constraints. An observation $O$ agrees with $\mathscr{P}$ and $\mathscr{C}$ if (1) $\operatorname{sem}_{S}(\mathscr{P}) \neq \emptyset\left(\mathscr{P}\right.$ is consistent); (2) $\mathscr{P} \models_{S} \mathscr{C}$; and (3) $\mathscr{P} \models_{S} O$.

To get this notion of agreement, it is necessary that the program be consistent and skeptically entail the observation. But, in addition, the program must also satisfy the integrity constraints in a very strong sense. Namely, all its models must satisfy them.

Definition 3 (AGREEMENT B (DENECKER AND KAKAS 2002))

Let $\mathscr{P}$ be a program and $\mathscr{C}$ a set of integrity constraints. An observation $O$ agrees with $\mathscr{P}$ and $\mathscr{C}$ if (1) $\mathscr{P} \models_{S} O$; and (2) there is $M \in \operatorname{sem}_{S}(\mathscr{P})$ such that $M \models \mathscr{C}$.

This definition is a relaxation of the previous one. To get this notion of agreement, at least one model of the program must satisfy the integrity constraints (not all, as before, and here is where the conditions are relaxed). This, in particular, means that the program is consistent. Moreover, exactly as before, the program must skeptically entail the observation.

\section{Definition 4 (AgREEMENT C)}

Let $\mathscr{P}$ be a program and $\mathscr{C}$ a set of integrity constraints. An observation $O$ agrees with $\mathscr{P}$ and $\mathscr{C}$ if for every $M \in \operatorname{sem}_{S}(\mathscr{P})$ such that $M \models \mathscr{C}, M \models O$.

Here to have an agreement, as in Definition 3. we require that at least one model of the program satisfies the integrity constraints and we weaken the other condition. Namely, we now only require that the observation holds in every model of the program satisfying the integrity constraints and not in every model of the programs.

Definition 5 (AGREEMENT D (BARAL AND GELFOND 1994))

Let $\mathscr{P}$ be a program and $\mathscr{C}$ a set of integrity constraints. An observation $O$ agrees with $\mathscr{P}$ and $\mathscr{C}$ if there is $M \in \operatorname{sem}_{S}(\mathscr{P})$ such that $M \models \mathscr{C}$ and $M \models O$.

This is the final relaxations of the conditions for an agreement. There must be models of a program that satisfy the integrity constraints (just as in Definition 4). But it is enough that one of such models (not all, as in Definition 4) satisfies the observation. Each of these types of agreement yields the corresponding notion of an explanation. 
Definition 6 (ABDuctive ExPLANATION)

Let $\mathscr{T}=\langle\mathscr{P}, \mathscr{A}, \mathscr{C}\rangle$ be an abductive theory and $O$ an observation. A pair $\Delta=(E, F)$, where $E$ and $F$ are disjoint finite sets of abducibles and $F \subseteq \mathscr{P}$, is an explanation (of type $A, B, C$, and $D$ ) if $O$ is in agreement (of type $A, B, C$, and $D$, respectively) with $\mathscr{P}^{\Delta}=(\mathscr{P} \cup E) \backslash F$ and $\mathscr{C}$. We denote the sets of explanations of each type by $\Psi_{A}(O, \mathscr{T}), \Psi_{B}(O, \mathscr{T}), \Psi_{C}(O, \mathscr{T})$ and $\Psi_{D}(O, \mathscr{T})$.

The observations made above about each next concept of an agreement being less restrictive, imply the following relationships between the corresponding notions of explanations.

Proposition 1

For every abductive theory $\mathscr{T}$ and an observation $O$,

$$
\Psi_{A}(O, \mathscr{T}) \subseteq \Psi_{B}(O, \mathscr{T}) \subseteq \Psi_{C}(O, \mathscr{T}) \subseteq \Psi_{D}(O, \mathscr{T})
$$

In general, the four concepts of explanations do not coincide. Which of them to choose may depend on a particular application domain. This issue is not of our concern here. Instead, we focus on the key challenge of abductive reasoning to find general principles that can narrow down a class of explanations and that are independent of what notion of an explanation is used. This is indeed an important problem as even in the case of explanations of type $A$ multiple explanations are possible and not all of them are equally compelling.

The two most commonly used principles are subset minimality and cardinality minimality. We say that an explanation $\Delta=(E, F)$ is subset minimal if there is no other explanation $\Delta^{\prime}=$ $\left(E^{\prime}, F^{\prime}\right)$ such that $E^{\prime} \subseteq E$ and $F^{\prime} \subseteq F$. Similarly, $\Delta=(E, F)$ is cardinality minimal if there is no explanation $\Delta^{\prime}=\left(E^{\prime}, F^{\prime}\right)$ such that $\left|E^{\prime}\right|+\left|F^{\prime}\right|<|E|+|F|$. We propose in the next section yet another general principle based on the degree of arbitrariness.

\section{How arbitrary is a solution?}

In this section we introduce the degree of arbitrariness in an explanation of an observation as a measure of how arbitrary the explanation is. Concepts introduced below apply to each of the four basic models of abductive reasoning, therefore we will use the generic term explanation without denoting its specific type. We start with examples to motivate our discussion.

Example 3

Let us consider the abduction problem presented in Example 2, and the explanations $\Delta_{u}=\left(S_{u}, \emptyset\right)$ and $\Delta_{d a n}=\left(E_{d a n}, \emptyset\right)$. In each explanation $\Delta_{u}$, the constant $u$ can be replaced with any other constant $u^{\prime}$ in the vocabulary and the result, $\Delta_{u^{\prime}}$, is also an explanation. That is, the occurrences of $u$ are not constrained by the program or, to put it differently, they are arbitrary. In contrast, replacing the constant $d a n$ in $\Delta_{d a n}$ with any other constant does not yield an explanation (assuming there are no other visitors in the program but dan). Thus, there is no arbitrariness in $\Delta_{d a n}$. In other words, $\Delta_{d a n}$ is constrained by the available information.

We will use the idea of "replaceability" to formalize the notions of arbitrariness and constrainedness, and their generalization, the degree of arbitrarines.

Definition 7 (OCCURRENCE)

Let $p(x)$ be a ground atom, where $p$ has arity $n$ and $k$ is an integer such that $1 \leq k \leq n$. We denote by $p(x)[k]$ the constant in the position $k$ in $p(x)$.

If $E$ is a set of ground atoms, an occurrence of a constant $c$ in $E$ is an expression of the form $p(x)^{k}$, where $p(x)$ is an atom in $E$, and $p(x)[k]=c$. 
Definition 8 (REPLACEMENT FUNCTION)

Let $\mathscr{T}=\langle\mathscr{P}, \mathscr{A}, \mathscr{C}\rangle$ be an abductive theory, $E$ a set of abducibles (that is, $E \subseteq \mathscr{A}^{\mathscr{D}}$ ), and let $c$ be a constant occurring in $E$. A replacement function for $E$ and $c$ determined by a non-empty set $C$ of some (not necessarily all) occurrences of $c$ in $E$, is a function $f_{E, C}: \mathscr{D} \rightarrow 2^{\mathscr{A} D}$ such that for each $x \in \mathscr{D}, f_{E, C}(x)$ is the set $E^{\prime}$ obtained by replacing with $x$ each constant $c$ in $E$ referred to by an occurrence in $C$.

We observe that, given a set $E$ and a constant $c$ occurring in $E$ in $n$ places, the number of possible replacement functions is $2^{n}-1$.

\section{Example 4}

Let us consider the set $E=\{p(1,2), s(2,3)\}$ and the constant 2. The possible replacement functions are: (1) $f_{E, C_{1}}$, where $C_{1}=\left\{p(1,2)^{2}\right\}$; (2) $f_{E, C_{2}}$, where $C_{2}=\left\{s(2,3)^{1}\right\}$; and (3) $f_{E, C_{3}}$, where $C_{3}=\left\{p(1,2)^{2}, s(2,3)^{1}\right\}$.

\section{Definition 9 (INDEPENDENCE OF REPLACEMENT FUNCTIONS)}

Let $f_{E, C_{1}}$ and $f_{E, C_{2}}$ be replacement functions for a set $E \subseteq \mathscr{A}^{\mathscr{D}}$ and for constants $c_{1}$ and $c_{2}$, respectively. We say that $f_{E, C_{1}}$ and $f_{E, C_{2}}$ are independent if $c_{1} \neq c_{2}$ or if $C_{1} \cap C_{2}=\emptyset$.

Let us consider the replacement functions presented in Example 4 . While the functions $f_{E, C_{1}}$ and $f_{E, C_{2}}$ are independent, the functions $f_{E, C_{3}}$ and $f_{E, C_{1}}$ (similarly, $f_{E, C_{3}}$ and $f_{E, C_{2}}$ ) are not independent.

\section{Definition 10 (DEGREE OF ARBITRARINESS)}

Let $\mathscr{T}=\langle\mathscr{P}, \mathscr{A}, \mathscr{C}\rangle$ be an abductive theory, $O$ an observation, $\Delta=(E, F)$ an explanation for $O$ wrt $\mathscr{T}$, and let $\xi$ be an arbitrary constant in $\mathscr{D}$ not occurring in $E \cup O \cup \mathscr{P}$. The degree of arbitrariness of $\Delta$, denoted $\delta(\Delta)$, is the maximum number of pairwise independent replacement functions $f_{E, C}$ (not necessarily all for the same constant) such that $\Delta^{\prime}=\left(f_{E, C}(\xi), F\right)$ is an explanation for $O$ wrt $\mathscr{T}$.

Since the domain $\mathscr{D}$ is infinite, one can always find a constant $\xi$ not occurring in $E \cup O \cup \mathscr{P}$. Moreover, since $E$ is finite, there is an upper bound on the number of pairwise independent replacement functions one can have for $E$. Finally, the specific choice of the replacement constant $\xi$ does not affect the maximum. Thus, the degree of arbitrariness is well defined. The examples below illustrate the concepts we have introduced above.

\section{Example 5}

Let $\mathscr{T}=\langle\mathscr{P}, \mathscr{A}, \emptyset\rangle$, where the program $\mathscr{P}$ consists of the facts $\{p(1), p(2), q(1), q(2), q(3)\}$ and of a single rule $t \leftarrow p(X)$, not $q(X)$. Let us suppose that $p$ and $q$ are abducible predicates and that $O=\{t\}$. The following pairs of sets of abducibles form explanations for $O$ wrt $\mathscr{T}$ :

$\Delta_{1}=(\emptyset,\{q(1)\}) . \Delta_{2}=(\emptyset,\{q(2)\}) . \Delta_{3}=(\{p(3)\},\{q(3)\}) . \Delta_{x}=(\{p(x)\}, \emptyset)$, where $x \notin\{1,2,3\}$.

It is evident that $\delta\left(\Delta_{1}\right)=\delta\left(\Delta_{2}\right)=0$. Similarly, it is evident that $\delta\left(\Delta_{3}\right)=\delta\left(\Delta_{x}\right)=1$ (the only constant in the "add" part of these explanations can be replaced with a fresh constant $\xi$ and the result is an explanation).

In Example 5, the explanation $\Delta_{3}$ is not satisfactory. Once we decide to remove $q(3)$, there is no reason why we have to add $p(3)$. Adding any atom $p(\xi)$, with $\xi \notin\{1,2\}$ works equally well. Thus, the choice of the constant 3 in $p(3)$ is arbitrary and not grounded in the information available in the theory. Similarly, $\Delta_{x}$, where $x \notin\{1,2,3\}$ is not satisfactory either. Here too, the choice of $x$ is not grounded in the abductive theory and the observation. The explanations $\Delta_{1}$ and $\Delta_{2}$ do not show this arbitrariness. 


\section{Example 6}

Let $\mathscr{T}=\langle\mathscr{P}, \mathscr{A}, \emptyset\rangle$, where $\mathscr{A}=\{q, r, t\}$ and $\mathscr{P}$ consists of the facts $q(a, b), q(a, c)$, and $r(a, b, c)$, and two rules $p(X) \leftarrow q(X, Y), s(X, Y, Z)$, and $s(X, Y, Z) \leftarrow r(X, Y, Z), t(X, Z)$. Let us suppose $O=$ $\{p(a)\}$. One can check that each of the following pairs of sets of abducibles is an explanation:

$$
\begin{aligned}
& \Delta_{x_{1}, x_{2}}=\left(\left\{q\left(a, x_{1}\right), r\left(a, x_{1}, x_{2}\right), t\left(a, x_{2}\right)\right\}, \emptyset\right), \text { where } x_{1} \neq c, x_{1} \neq b \text { and } x_{2} \neq c \\
& \Delta_{x_{3}}=\left(\left\{r\left(a, b, x_{3}\right), t\left(a, x_{3}\right)\right\}, \emptyset\right), \text { where } x_{3} \neq c \\
& \Delta=(\{t(a, c)\}, \emptyset) .
\end{aligned}
$$

It is evident that if $x_{1} \neq x_{2}$, then $\delta\left(\Delta_{x_{1}, x_{2}}\right)=2$. Indeed changing all occurrences of $x_{1}$ or all occurrences of $x_{2}$ to a new constant $\xi$ results in an explanation. In addition, the corresponding replacement functions for each constant and all its occurrences are obviously independent (they concern different constants). Finally, replacing either constant in only one position does not yield an explanation. More interestingly, if $x_{1}=x_{2}=x, \delta\left(\Delta_{x, x}\right)=2$, too. Here, $x$ has four occurrences in $\Delta_{x, x}: q(a, x)^{2}, r(a, x, x)^{2}, r(a, x, x)^{3}$ and $t(a, x)^{2}$. Let $f_{1}$ and $f_{2}$ be the replacement functions for $x$ determined by the first two and the last two positions, respectively. Then $f_{1}$ and $f_{2}$ are independent, and both $\left(f_{1}(\xi), \emptyset\right)$ and $\left(f_{2}(\xi), \emptyset\right)$ are explanations. However, three mutually independent functions with this property cannot be found. Similarly, one can see that $\delta\left(\Delta_{x_{3}}\right)=1$ (all occurrences of $x_{3}$ are free for a simultaneous change) and $\delta(\Delta)=0$ (neither $a$ nor $c$ can be changed to a fresh constant).

In this example, all explanations are minimal and so, the subset-minimality criterion is not discriminating enough. However, $\Delta$ arguably is more compelling than the other ones. It uses no extraneous constants, and all constants occurring in it are constrained by the theory and an observation. The lowest degree of arbitrariness criterion correctly identifies $\Delta$ as the only preferred explanation. We will refer to it as the principle of minimum arbitrariness.

The most compelling are those explanations that have no arbitrariness at all. This suggests the notion of constrained explanations.

\section{Definition 11 (CONSTRAINEd AND ARBITRARY EXPlanATIONS)}

Let $\mathscr{T}$ be an abductive theory $\langle\mathscr{P}, \mathscr{A}, \mathscr{C}\rangle, O$ an observation, and $\Delta$ an explanation for $O$ wrt $\mathscr{T}$. We say that $\Delta$ is constrained if $\delta(\Delta)=0$. Otherwise, $\Delta$ is arbitrary.

In the remainder of this paper we discuss the principle of the lowest degree of arbitrariness, focusing our study primarily on the class of constrained explanations. We start with some general observations.

The principle of minimum arbitrariness can be used with all types of explanations we discussed in the previous sections. Moreover, it is "orthogonal" to other criteria one might consider when selecting preferred explanations such as the subset or cardinality minimality. Therefore, it can be combined with them. For instance, we might consider as preferred those subset-minimal explanations that have the smallest degree of arbitrariness (if we believe, that subset minimality is more important than minimum arbitrariness). For instance, coming back to Example 2, we see that the explanations $\left(E_{\text {tom }}, \emptyset\right),\left(E_{\text {mary }}, \emptyset\right),\left(S_{u}, \emptyset\right), u \neq$ tom, $\left(E_{\text {dan }}, \emptyset\right)$, and $\left(V_{u}, \emptyset\right), u \neq d a n$, are subset minimal. Selecting from among them only the constrained ones yields $\left(E_{\text {tom }}, \emptyset\right),\left(E_{\text {mary }}, \emptyset\right)$, and $\left(E_{d a n}, \emptyset\right)$. Alternatively, we could take as preferred those explanations with the minimum degree of arbitrariness that are subset minimal (if we believe that minimum arbitrariness should be the primary consideration). In Example 2, the explanation $\left(E_{t o m, d a n}, \emptyset\right)$ is constrained but not minimal. In general, the two concepts are different. Similar examples can be provided for the minimum cardinality criterion. We do not consider these and other possible combinations of 
the principles in this paper but focus exclusively on the properties of the principle of minimum arbitrariness.

The degree of arbitrariness of an explanation $(E, F)$ depends only on the "add" part $E$; the "delete" component, $F$, has no effect on arbitrariness. Intuitively, the reason is that we can delete only those atoms that are in $\mathscr{P}$. Thus, if we replace a constant in an atom $p$ in $F$ with a fresh constant $\xi$, the effect simply is that $p$ is no longer deleted. The same effect can be achieved by considering $F \backslash\{p\}$ in place of $F$. It is natural to impose on $F$ some requirements, such as subset or cardinality minimality. However, as we noted above, we do not pursue this possibility here.

The next result shows that constrained explanations use only constants that are mentioned in an abductive theory and an observation. This property is consistent with the general principle of parsimony (Occam's razor). It is important as it allows us to restrict the scope of searches for constrained explanations 2

\section{Theorem 1}

Let $\mathscr{T}=\langle\mathscr{P}, \mathscr{A}, \mathscr{C}\rangle$ be an abductive theory and $(E, F)$ a constrained explanation of an observation $O$. Then every constant symbol occurring in $(E, F)$ occurs in $\mathscr{T}$ or in $O$.

Finally, we note that the minimum arbitrariness criterion does not impose any conditions on abducible predicates of arity 0 and some other criteria should be considered. Therefore, we are primarily interested in the case when every abducible predicate symbol has a positive arity.

\section{Computational Complexity}

Our primary technical contribution concerns reasoning about constrained explanations. We are interested in the following two problems: deciding whether a given explanation is constrained; and deciding whether a constrained explanation exists. We restrict attention to abductive theories with stratified programs and assume that these programs are interpreted by the stable-model semantics. This is an important class of abductive theories. First, stratified programs are regarded as semantically "non-controversial." Indeed, the stable-model semantics and the well-founded semantics coincide on stratified programs and are generally accepted as providing them the correct meaning. Second, under the stable-model semantics, for abductive theories with stratified programs, the distinctions between the four types of explanations we introduced disappear. Formally, we have the following result.

\section{Theorem 2}

For every abductive theory $\mathscr{T}=\langle\mathscr{P}, \mathscr{A}, \mathscr{C}\rangle$, where $\mathscr{P}$ is stratified and interpreted under the stable-model semantics, and every observation $O$,

$$
\Psi_{A}(O, \mathscr{T})=\Psi_{B}(O, \mathscr{T})=\Psi_{C}(O, \mathscr{T})=\Psi_{D}(O, \mathscr{T}) .
$$

Because of Theorem 2, below we use the generic term explanation without specifying its type.

Let $\langle\mathscr{P}, \mathscr{A}, \mathscr{C}\rangle$ be an abductive logic theory. We represent $\mathscr{P}$ as the union of a set $B$ of all abducibles in $\mathscr{P}$ and the set $R$ of the remaining facts and rules. We will consider the complexity of the problems stated above under the assumption that $R$ and $\mathscr{C}$ are fixed and input consists of $B$ and an observation $O$. We start our study of the complexity by noting the following two upper bounds.

\footnotetext{
${ }^{2}$ The proofs of all results we give in the paper can be found in the appendix
} 


\section{Theorem 3}

Let $\mathscr{A}$ be a set of abducible predicates, $R$ a (fixed) stratified program with no abducible predicates in the heads of its rules, and $\mathscr{C}$ a (fixed) set of integrity constraints.

1. The following problem is in coNP: given a set $B$ of abducibles, an observation $O$, and a pair $\Delta=(E, F)$ of sets of abducibles, decide whether $\Delta$ is a constrained explanation for $O$ wrt $\langle R \cup B, \mathscr{A}, \mathscr{C}\rangle$.

2. The following problem is in $\Sigma_{2}^{P}$ : given a set $B$ of abducibles and an observation $O$, decide whether a constrained explanation for $O$ wrt $\langle R \cup B, \mathscr{A}, \mathscr{C}\rangle$ exists.

There are three sources of complexity: negation in programs, recursion in programs, and the presence of integrity constraints. The next three results show that each of these sources by itself pushes the complexity up to match the upper bounds of Theorem 3 . The first of them concerns the case when the program in an abductive theory is non-recursive but with negation, and there are no integrity constraints.

\section{Theorem 4}

Let $\mathscr{A}$ be a set of abducible predicates and $R$ a (fixed) non-recursive program with no abducible predicates in the heads of its rules.

1. The following problem is coNP-complete: given a set $B$ of abducibles, an observation $O$, and a pair $(E, F)$ of sets of abducibles, decide whether $(E, F)$ is a constrained explanation for $O$ wrt $\langle R \cup B, \mathscr{A}, \emptyset\rangle$.

2. The following problem is $\Sigma_{2}^{P}$-complete: given a set $B$ of abducibles and an observation $O$, decide whether a constrained explanation for $O$ wrt $\langle R \cup B, \mathscr{A}, \emptyset\rangle$ exists.

The next theorem shows that when there are no integrity constraints, disallowing negation, that is, restricting attention to Horn programs, also leads to the same complexity results as long as we allow recursion.

\section{Theorem 5}

Let $\mathscr{A}$ be a set of abducible predicates and $R$ a (fixed) Horn program with no abducible predicates in the heads of its rules.

1. The following problem is coNP-complete: given a set $B$ of abducibles, an observation $O$, and a pair $(E, F)$ of sets of abducibles, decide whether $(E, F)$ is a constrained explanation for $O$ wrt $\langle R \cup B, \mathscr{A}, \emptyset\rangle$.

2. The following problem is $\Sigma_{2}^{P}$-complete: given a set $B$ of abducibles and an observation $O$, decide whether a constrained explanation for $O$ wrt $\langle R \cup B, \mathscr{A}, \emptyset\rangle$ exists.

The third result addresses the case of abductive theories with particularly simple programs, namely, non-recursive Horn, but with integrity constraints.

\section{Theorem 6}

Let $\mathscr{A}$ be a set of abducible predicates, $R$ a (fixed) non-recursive Horn program with no abducible predicates in the heads of its rules, and $\mathscr{C}$ a (fixed) set of integrity constraints.

1. The following problem is coNP-complete: given a set $B$ of abducibles, an observation $O$, and a pair $(E, F)$ of sets of abducibles, decide whether $(E, F)$ is a constrained explanation for $O$ wrt $\langle R \cup B, \mathscr{A}, \mathscr{C}\rangle$. 
2. The following problem is $\Sigma_{2}^{P}$-complete: given a set $B$ of abducibles and an observation $O$, decide whether a constrained explanation for $O$ wrt $\langle R \cup B, \mathscr{A}, \mathscr{C}\rangle$ exists.

On the other end of the spectrum, we have a particularly simple case when neither of the three sources of complexity is present: the case of abductive theories with non-recursive Horn programs and without integrity constraints. For this class of theories the two problems are tractable.

\section{Theorem 7}

Let $\mathscr{A}$ be a set of abducible predicates and $R$ a (fixed) non-recursive Horn program with no abducible predicates in the heads of its rules. The following problems are in $\mathrm{P}$.

1. Given a set $B$ of abducibles, an observation $O$, and a pair $(E, F)$ of sets of abducibles, decide whether $(E, F)$ is a constrained explanation for $O$ wrt $\langle R \cup B, \mathscr{A}, \emptyset\rangle$.

2. Given a set $B$ of abducibles and an observation $O$, decide whether a constrained explanation for $O$ wrt $\langle R \cup B, \mathscr{A}, \emptyset\rangle$ exists.

\section{Related Work}

Abduction was introduced to artificial intelligence in early 1970s by Harry Pople Jr. (1973), where it is now commonly understood as the inference to the best explanation (Josephson and Josephson 1996). Over the years several criteria have been proposed to identify the preferred (best) explanations, all rooted in the Occam's razor parsimony principle. The most commonly considered one is subsetminimality (Bylander et al. 1991, Konolige 1992; Selman and Levesque 1990). A more restrictive condition of minimum cardinality has also been broadly studied (Peng and Reggia 1990). The abduction reasoning formalism we study in the paper uses logic programs to represent background knowledge in abductive theories. It is referred to as abductive logic programming (Eshghi and Kowalski 1989, Kakas et al. 1992, Dung 1991). Abductive explanations which allow the removal of hypotheses are first introduced by Inoue and Sakama (1995). The importance of abductive logic programming to knowledge representation was argued by Denecker and Schreye (1995). It was applied in diagnosis (Console et al. 1996), planning (Eshghi 1988, do Lago Pereira and de Barros 2004), natural language understanding (Balsa et al. 1995), and casebased reasoning (Satoh 1996). Denecker and Kakas (2002) provide a comprehensive survey of the area.

The complexity of abductive reasoning was first studied by Bylander et al. (1991). Eiter et al. (1997) studied the complexity of reasoning tasks in the abductive logic programming setting.

The profusion of abductive explanations was explicitly noted by Maher (2005) in his work on constraint abduction. Maher considers a differennt setting and handles the problem by different techniques. The key similarity is that in Maher's setting, as in ours, symbols from the vocabulary not present in the theory can give rise to alternative explanations.

None of the earlier works on abduction considered the concepts of constrainedness or arbitrariness. These concepts were proposed by us for the setting of view updates in deductive databases (Caroprese et al. 2012). View updating consists of modifying base relations to impose properties on view relations, that is, relations defined on the database by queries. The subject has received much attention in database research (cf. the survey papers by Fraternali and Paraboschi (1993), and Mayol and Teniente (1999)). There is a natural connection between view updating and abduction (Kakas and Mancarella 1990a; Console et al. 1995). The view plays the role of the background theory, all base relation symbols are abducible predicates, and requests for a view 
update are observations. The role of integrity constraints is the same in both areas. However, there is an important distinction here. In view updating, integrity constraints concern only base relation, a restriction not present in abductive settings. Our present work adapts the notions of constrainedness and arbitrariness to the more general setting of abduction. Importantly, we introduce the new concept of the degree of arbitrariness, which allows us to compare explanations even when no constrained explanations exist.

\section{Concluding Remarks}

We proposed a new approach to limiting the space of explanations in abductive logic programming extended by integrity constraints. Specifically, we introduced the degree of arbitrariness as a measure of the quality of an explanation. It imposes a hierarchy on the space of explanations (possibly already narrowed down by means of other principles): the lower the degree of arbitrariness, the more compelling an explanation. Explanations with the degree of arbitrariness equal to 0 are particularly important. We presented a detailed account of the complexity of reasoning with constrained explanations when programs in abductive theories are stratified. In our discussion in Sections 2 and 3 we were implicitly assuming that the set $\operatorname{sem}_{S}(P)$ consisted of two-valued interpretations. However, our definitions can also be extended to the three-valued well-founded semantics. Moreover, since for stratified programs the well-founded and the stable-model semantics coincide, all complexity results we obtained hold for that case, too. Finally, our approach applies to each of the four basic models of abductive reasoning, three of which have been studied before, with the remaining one (Definition 4 ) to the best of our knowledge being new. Our discussion and the results suggest that the notions of the degree of arbitrariness and constrainedness are important additions to the space of fundamental principles of abductive reasoning. We note, however, that as with other principles there are situations where the minimum degree of arbitrariness may not be the appropriate principle to use for abduction. For example, let us consider the transitive closure program containing two rules: $t c(x, y) \leftarrow r(x, y)$ and $t c(x, y) \leftarrow r(x, z), t c(z, y)$ with an abducible predicate $r$. If we observe $t c(a, b)$, the explanation $E_{1}=(\{r(a, b)\}, \emptyset)$ is constrained while $\left.E_{2}=(\{r(a, z), r(z, b)\}, \emptyset)\right\}$ is arbitrary ( $z$ can be replaced by any constant in the vocabulary of the language). Thus, according to the minimum degree of arbitrariness criterion, $E_{1}$ is preferred to $E_{2}$. However, if the relation $t c$ is the ancestor relation, $r$ is the parent relation and ancestor $(a, b)$ is observed, preferring the constrained explanation $\{$ parent $(a, b)\}$ to $\{$ parent $(a, c)$, parent $(c, b)\}$, which is arbitrary, may be a point of dispute. Establishing conditions to help decide which criteria to use when is a grand challenge of the area of abduction. For the example we just gave, we note that the minimum cardinality principle would give the same result as the minimum arbitrariness one and would be open to the same question. Our work opens several avenues for future research. First, we do not have a clear picture of the complexity of the case of abductive theories with non-recursive Horn programs and with integrity constraints restricted only to abducible predicates. This case is of interest due to its view updating applications. Next, there is a challenging problem of resolving the complexity of reasoning with constrained explanations in the case of abductive theories with arbitrary programs.

\section{Acknowledgments}

The third author was supported by the NSF grant IIS-0913459. 


\section{References}

Balsa, J., Dahl, V., And Lopes, J. G. P. 1995. Datalog Grammars for Abductive Syntactic Error Diagnosis and Repair. In Proceedings of the Natural Language Understanding and Logic Programming Workshop, Lisbon, 1995. 111-125.

Baral, C. And Gelfond, M. 1994. Logic Programming and Knowledge Representation. J. Log. Program. 19/20, 73-148.

Bylander, T., Allemang, D., Tanner, M. C., And Josephson, J. R. 1991. The Computational Complexity of Abduction. Artificial Intelligence 49, 1-3, 25-60.

Caroprese, L., Trubitsyna, I., Truszczynski, M., and Zumpano, E. 2012. The View-update Problem for Indefinite Databases. In Proceedings of the 13th European Conference on Logics in Artificial Intelligence, JELIA 2012, L. F. del Cerro, A. Herzig, and J. Mengin, Eds. Lecture Notes in Computer Science, vol. 7519. Springer, 134-146.

Console, L., Portinale, L., And Dupré, D. T. 1996. Using Compiled Knowledge to Guide and Focus Abductive Diagnosis. IEEE Trans. Knowl. Data Eng. 8, 5, 690-706.

Console, L., Sapino, M. L., And Dupré, D. T. 1995. The Role of Abduction in Database View Updating. J. Intell. Inf. Syst. 4, 3 (May), 261-280.

Denecker, M. And KaKas, A. C. 2002. Abduction in Logic Programming. In Computational Logic: Logic Programming and Beyond, Essays in Honour of Robert A. Kowalski, A. C. Kakas and F. Sadri, Eds. Lecture Notes in Computer Science, vol. 2407. Springer, 402-436.

Denecker, M. And Schreye, D. D. 1995. Representing Incomplete Knowledge in Abductive Logic Programming. J. Log. Comput. 5, 5, 553-577.

Do Lago Pereira, S. AND DE BArros, L. N. 2004. Planning with Abduction: A Logical Framework to Explore Extensions to Classical Planning. In Proceedings of the 17th Brazilian Symposium on Artificial Intelligence, SBIA 2004, A. L. C. Bazzan and S. Labidi, Eds. Lecture Notes in Computer Science, vol. 3171. Springer, 62-72.

DunG, P. M. 1991. Negations as Hypotheses: An Abductive Foundation for Logic Programming. In Proceedings of the 8th International Conference on Logic Programming, K. Furukawa, Ed. MIT Press, 3-17.

Eiter, T., Gottlob, G., And Leone, N. 1997. Abduction from Logic Programs: Semantics and Complexity. Theor. Comput. Sci. 189, 1-2, 129-177.

Eshghi, K. 1988. Abductive Planning with Event Calculus. In Proceedings of the 5th International Conference and Symposium on Logic Programming, R. A. Kowalski and K. A. Bowen, Eds. MIT Press, $562-579$.

Eshghi, K. AND Kowalski, R. A. 1989. Abduction Compared with Negation by Failure. In Proceedings of the 6th International Conference on Logic Programming, G. Levi and M. Martelli, Eds. MIT Press, 234-254.

Fraternali, P. AND Paraboschi, S. 1993. A Review of Repairing Techniques for Integrity Maintenance. In Proceedings of the 1st International Workshop on Rules in Database Systems, N. W. Paton and M. H. Williams, Eds. Workshops in Computing. Springer, 333-346.

Gelfond, M. And Lifschitz, V. 1988. The Stable Semantics for Logic Programs. In Proceedings of the 5th International Conference and Symposium on Logic Programming, R. A. Kowalski and K. A. Bowen, Eds. MIT Press, 1070-1080.

InOUE, K. AND SAKAMA, C. 1995. Abductive Framework for Nonmonotonic Theory Change. In Proceedings of the 14th International Joint Conference on Artificial Intelligence, IJCAI 95. Morgan Kaufmann, 204-210.

Josephson, J. And Josephson, S. 1996. Abductive Inference: Computation, Philosophy, Technology. Cambridge University Press.

Kakas, A. C., Kowalski, R. A., And Toni, F. 1992. Abductive Logic Programming. J. Log. Comput. 2, 6, 719-770.

Kakas, A. C. And Mancarella, P. 1990a. Database updates through abduction. In Proceedings of the 
16th International Conference on Very Large Data Bases, VLDB 1990, D. McLeod, R. Sacks-Davis, and H.-J. Schek, Eds. Morgan Kaufmann, 650-661.

KaKas, A. C. And Mancarella, P. 1990b. Generalized stable models: A semantics for abduction. In Proceedings of the 9th European Conference on Artificial Intelligence, ECAI 1990, L. Aiello, Ed. Pitman, London/Boston, 385-391.

Konolige, K. 1992. Abduction Versus Closure in Causal Theories. Artificial Intelligence 53, 2-3, 255272.

MAHer, M. J. 2005. Herbrand constraint abduction. In Proceedings of the 20th IEEE Symposium on Logic in Computer Science, LICS 2005. IEEE Computer Society, 397-406.

MareK, W. And Subrahmanian, V. 1992. The Relationship Between Stable, Supported, Default and Autoepistemic Semantics for General Logic Programs. Theoretical Computer Science 103, 2, 365-386.

Mayol, E. And Teniente, E. 1999. A Survey of Current Methods for Integrity Constraint Maintenance and View Updating. In Advances in Conceptual Modeling, Proceedings of ER '99 Workshops on Evolution and Change in Data Management, Reverse Engineering in Information Systems, and the World Wide Web and Conceptual Modeling, P. P. Chen, D. W. Embley, J. Kouloumdjian, S. W. Liddle, and J. F. Roddick, Eds. Lecture Notes in Computer Science, vol. 1727. Springer, 62-73.

PeIRCE, C. 1995. Abduction and Induction. In Philosophical Writing of Peirce, J. Buchler, Ed. Dover, New York, Chapter 11.

Peng, Y. And Reggia, J. 1990. Abductive Inference Models for Diagnostic Problem Solving. Springer.

Pople, H. E. 1973. On the Mechanization of Abductive Logic. In Proceedings of the 3rd International Joint Conference on Artificial Intelligence, IJCAI 1973, N. J. Nilsson, Ed. William Kaufmann, 147-152.

SATOH, K. 1996. Translating Case-Based Reasoning into Abductive Logic Programming. In Proceedings of the 12th European Conference on Artificial Intelligence, ECAI 1996, W. Wahlster, Ed. John Wiley and Sons, Chichester, UK, 142-146.

Selman, B. And Levesque, H. J. 1990. Abductive and Default Reasoning: A Computational Core. In Proceedings of the 8th National Conference on Artificial Intelligence, AAAI 1990, H. E. Shrobe, T. G. Dietterich, and W. R. Swartout, Eds. 343-348.

VAn Gelder, A., Ross, K., And Schlipf, J. 1991. The Well-Founded Semantics for General Logic Programs. Journal of the ACM 38, 3, 620-650. 


\section{Appendix - Proofs}

\section{Theorem 1}

Let $\mathscr{T}=\langle\mathscr{P}, \mathscr{A}, \mathscr{C}\rangle$ be an abductive theory and $(E, F)$ a constrained explanation of an observation $O$. Then every constant symbol occurring in $(E, F)$ occurs in $\mathscr{T}$ or in $O$.

\section{Proof}

Since $F \subseteq \mathscr{P}$ (as required by the definition of an explanation), every constant occurring in $F$ occurs in $\mathscr{P}$. If $\alpha$ is a constant appearing in $E$ but not in $\mathscr{T}$ nor in $O$, then changing $\alpha$ to a fresh constant $\xi$ results in an explanation 3 and so, contradicts the constrainedness of $(E, F)$.

\section{Theorem 2}

For every abductive theory $\mathscr{T}=\langle\mathscr{P}, \mathscr{A}, \mathscr{C}\rangle$, where $\mathscr{P}$ is stratified and interpreted under the stable-model semantics, and for every observation $O$,

$$
\Psi_{A}(O, \mathscr{T})=\Psi_{B}(O, \mathscr{T})=\Psi_{C}(O, \mathscr{T})=\Psi_{D}(O, \mathscr{T})
$$

Proof

The assertion follows by the fact that a stratified program admits exactly one stable model.

\section{Theorem 3}

Let $\mathscr{A}$ be a set of abducible predicates, $R$ a (fixed) stratified program with no abducible predicates in the heads of its rules, and $\mathscr{C}$ a (fixed) set of integrity constraints.

1. The following problem is in coNP: given a set $B$ of abducibles, an observation $O$, and a pair $\Delta=(E, F)$ of sets of abducibles, decide whether $\Delta$ is a constrained explanation for $O$ wrt $\langle R \cup B, \mathscr{A}, \mathscr{C}\rangle$.

2. The following problem is in $\Sigma_{2}^{P}$ : given a set $B$ of abducibles and an observation $O$, decide whether a constrained explanation for $O$ wrt $\langle R \cup B, \mathscr{A}, \mathscr{C}\rangle$ exists.

\section{Proof}

(1) The complementary problem consists of deciding that $(E, F)$ is not an explanation or that is an arbitrary explanation. The following non-deterministic polynomial-time algorithm decides this problem. Since $R$ is stratified, one can compute the only stable model, say $M$, of $R \cup((B \cup E) \backslash F)$ in time linear in the size of $B$ and $(E, F)$. If $E$ and $F$ are not disjoint (which can be checked efficiently), or if $M \forall \forall O$, or if $M \forall \forall \mathscr{C}$, the $(E, F)$ is not an explanation. Otherwise, $(E, F)$ is an explanation and we proceed as follows. We non-deterministically guess the set $C$ of occurrences of some constant $c$ occurring in $E$. We then compute $\left(E^{\prime}, F\right)$ by replacing all occurrences of $c$ mentioned in $C$ with a fresh constant $\xi$ and, in the same way as before, determine whether $\left(E^{\prime}, F\right)$ is an explanation of $O$.

(2) If $(E, F)$ is a constrained explanation, then $E$ and $F$ consist of abducibles involving only constants appearing in $\mathscr{T}$ and $O$ (cf. Theorem 1 ). It follows, that if $(E, F)$ is a constrained explanation, the size of $E \cup F$ is polynomial in the size of the input. Thus, the problem can be decided by the following non-deterministic polynomial time algorithm with an oracle: guess sets $E$ and $F$ of abducibles and check that $(E, F)$ is a constrained explanation. By (1), that task can be decided by a call to a coNP oracle.

\footnotetext{
${ }^{3}$ We tacitly assume here that the semantics of logic programs we consider here are insensitive to the renaming of constants. All standard semantics of programs have this property.
} 


\section{Theorem 4}

Let $\mathscr{A}$ be a set of abducible predicates and $R$ a (fixed) non-recursive program with no abducible predicates in the heads of its rules.

1. The following problem is coNP-complete: given a set $B$ of abducibles, an observation $O$, and a pair $(E, F)$ of sets of abducibles, decide whether $(E, F)$ is a constrained explanation for $O$ wrt $\langle R \cup B, \mathscr{A}, \emptyset\rangle$.

2. The following problem is $\Sigma_{2}^{P}$-complete: given a set $B$ of abducibles and an observation $O$, decide whether a constrained explanation for $O$ wrt $\langle R \cup B, \mathscr{A}, \emptyset\rangle$ exists.

\section{Proof}

(1) The membership part was established in Theorem 3. Thus, it suffices to show the hardness part.

We note that the following version of the SAT problem is NP-complete (membership is evident, hardness follows by a straightforward reduction from SAT):

Input: A set of atoms $Y$ and a CNF formula $F$ over $Y$ that is not satisfied by the all-false assignment

Question: Is $F$ satisfiable (is the QBF formula $\exists Y F$ true)?

We will reduce that problem to the problem whether (under the notation in the statement of the theorem) an explanation $(E, F)$ is arbitrary.

Let then $Y$ be a set of atoms and $F$ a CNF theory that is not satisfied by the all-false assignment on $Y$. We denote by $C l(F)$ the set of clauses in $F$. Let us consider the vocabulary $\sigma$ consisting of predicate symbols bad $/ 0, i n_{Y} / 1$, clause $/ 1, p o s / 2$, gtd $/ 2$, choose $/ 2$, gate/1, true $/ 1$, holds $/ 1$, sometrue $/ 0$, allfalse $/ 0$, sat $/ 0$, clfalse $/ 0$ and goal $/ 0$, and an abductive theory

$$
\mathscr{T}(F)=\langle T(F),\{\text { choose }\}, \emptyset\rangle,
$$

where $T(F)=R \cup B, B$ consists of the atoms

1. $\operatorname{in}_{Y}(a)$, for every $a \in Y$

2. gate $(0)$, where $0 \notin Y$

3. $\operatorname{pos}(a, c)$, for every atom $a \in Y$ and clause $c \in C l(F)$ such that $a$ occurs non-negated in $c$

4. ngtd $(a, c)$, for every atom $a \in A t(F)$ and clause $c \in C l(F)$ such that $a$ occurs negated in $c$

and $R$ consists of the rules

1. clause $(C) \leftarrow \operatorname{pos}(A, C)$

2. $\operatorname{clause}(C) \leftarrow \operatorname{ngtd}(A, C)$

3. $\operatorname{true}(A) \leftarrow \operatorname{in}_{Y}(A)$, gate $(W)$, not $\operatorname{choose}(A, W)$

4. holds $(C) \leftarrow \operatorname{pos}(A, C)$, true $(A)$

5. holds $(C) \leftarrow \operatorname{ngtd}(A, C)$, not true $(A)$

6. clfalse $\leftarrow$ clause $(C)$, not holds $(C)$

7. sat $\leftarrow$ not clfalse

8. sometrue $\leftarrow \operatorname{in}_{Y}(A)$, true $(A)$

9. allfalse $\leftarrow$ not sometrue

10. bad $\leftarrow \operatorname{choose}(A, W)$, not $\operatorname{in}_{Y}(A)$

11. goal $\leftarrow$ allfalse, not $b a d$

12. goal $\leftarrow$ sat, not $b a d$. 
Let $\{$ goal $\}$ be the set of observed atoms. It is clear that $U=(\{\operatorname{choose}(a, 0): a \in Y\}, \emptyset)$ is an explanation (goal is derived through the first of its two rules). If $F$ is satisfiable, then let $Y^{\prime} \subseteq Y$ be (the representation of) an assignment that satisfies $F$. One can check that (\{choose $(a, 0): a \in$ $\left.\left.\left(Y \backslash Y^{\prime}\right)\right\} \cup\left\{\operatorname{choose}(a, \xi): a \in Y^{\prime}\right\}, \emptyset\right)$ is an explanation (now, goal can be derived via its second rule). Moreover, $Y^{\prime} \neq \emptyset$ (by our restriction on the class of formulas). Thus, (\{choose $(a, 0): a \in$ $Y\}, \emptyset)$ is arbitrary.

Conversely, let us assume that $U=(\{\operatorname{choose}(a, 0): a \in Y\}, \emptyset)$ is arbitrary. Then replacing some occurrences of one of the constants must yield an explanation. Replacing a constant $a \in Y$ with fresh constant symbol $\xi$ does not yield an explanation. Indeed, we would have $\operatorname{choose}(\xi, 0)$ and no $\operatorname{in}_{Y}(\xi)$ in the "add" part of the explanation. Thus, bad would hold and would block any possibility of deriving goal. It follows that one or more occurrences of 0 can be replaced by $\xi$ so that the result, $\left(\left\{\operatorname{choose}(a, 0): a \in\left(Y \backslash Y^{\prime}\right)\right\} \cup\left\{\operatorname{choose}(a, \xi): a \in Y^{\prime}\right\}, \emptyset\right)$, is an explanation of goal. Here $Y^{\prime} \subseteq Y$ is the non-empty set of elements in $Y$ identifying the occurrences of 0 replaced by $\xi$. Since $Y^{\prime} \neq \emptyset$, goal is derived via the second rule. It follows that sat is derivable and so, every clause in $F$ holds in the interpretation that assigns true to all elements of $Y^{\prime}$ and false to all other elements of $Y$. Thus, $F$ is satisfiable.

It follows that deciding whether an explanation $(E, F)$ is arbitrary is NP-hard. Since every explanation is either arbitrary or constrained, the problem to decide whether $(E, F)$ is constrained is coNP-hard.

(2) As before, the membership part of the assertion follows from Theorem 3, To prove the hardness part, we note that the following problem is $\Sigma_{2}^{P}$-complete:

Input: Two disjoint sets $X$ and $Y$ of atoms, and a DNF formula $G$ over $X \cup Y$ such that for every truth assignment $v_{X}$ to atoms in $X$, the all-false assignment to atoms in $Y$ is a model of formula $\left.G\right|_{\nu_{X}}$

Question: Is the quantified boolean formula $\Phi=\exists X \forall Y G$ true.

We will reduce it to our problem.

Let $F$ be the CNF obtained from $\neg G$ by applying the De Morgan's and the double negation laws. Clearly, $F \equiv \neg G$. Let $C l(F)$ be the set of clauses of $F$. Let us consider the vocabulary $\sigma$ consisting of predicate symbols $i_{X}, i n_{Y}$, clause $/ 1, p o s / 2$, ngtd $/ 2$, choose $/ 2$, gate $/ 1$, true $e_{X} / 1$, true $_{Y} / 1$, true $/ 1$, holds $/ 1$, sometrue $/ 0$, allfalse $/ 0$, sat $/ 0$, clfalse $/ 0, \mathrm{bad} / 0, \mathrm{good} / 1$, and goal $/ 0$, and an abductive theory

$$
\mathscr{T}(F)=\left\langle T(F),\left\{\text { true }_{X}, \text { choose }, \emptyset\right\rangle,\right.
$$

where $T(F)=R \cup B, B$ consists of the atoms:

1. $\operatorname{in}_{X}(a)$, for every $a \in X$

2. $\operatorname{in}_{Y}(a)$, for every $a \in Y$

3. $\operatorname{gate}(0)$, where $0 \notin Y$

4. $\operatorname{pos}(a, c)$, for every atom $a \in X \cup Y$ and clause $c \in C l(F)$ such that $a$ occurs non-negated in $c$

5. $\operatorname{ngtd}(a, c)$, for every atom $a \in X \cup Y$ and clause $c \in C l(F)$ such that $a$ occurs negated in $c$

and $R$ consists of the rules

1. $\operatorname{clause}(C) \leftarrow \operatorname{pos}(A, C)$

2. $\operatorname{clause}(C) \leftarrow \operatorname{ngtd}(A, C)$

3. $\operatorname{true}_{Y}(A) \leftarrow \operatorname{in}_{Y}(A), \operatorname{gate}(W)$, not choose $(A, W)$ 

4. $\operatorname{true}(A) \leftarrow \operatorname{true}_{X}(A)$
5. $\operatorname{true}(A) \leftarrow \operatorname{true}_{Y}(A)$
6. holds $(C) \leftarrow \operatorname{pos}(A, C)$, true $(A)$
7. holds $(C) \leftarrow \operatorname{ngtd}(A, C)$, not true $(A)$
8. clfalse $\leftarrow$ clause $(C)$, not holds $(C)$
9. sat $\leftarrow$ not clfalse
10. sometrue $\leftarrow \operatorname{in}_{Y}(A)$, true $_{Y}(A)$
11. allfalse $\leftarrow$ not sometrue
12. bad $\leftarrow \operatorname{choose}(A, W)$, not $\operatorname{in}_{Y}(A)$
13. bad $\leftarrow \operatorname{true}_{X}(A)$, not $\operatorname{in}_{X}(A)$
14. $\operatorname{good}(A) \leftarrow \operatorname{in}_{Y}(A), \operatorname{choose}(A, W)$
15. $\operatorname{bad} \leftarrow \operatorname{in}_{Y}(A), \operatorname{not} \operatorname{good}(A)$
16. goal $\leftarrow$ allfalse, not $b a d$
17. goal $\leftarrow$ sat, not bad.

Let $O=\{$ goal $\}$ be an observation. We will prove that $\Phi$ is true if and only if goal has a constrained explanation from $\mathscr{T}(F)$.

$(\Rightarrow)$ Let $v_{X}$ be an assignment of truth values to variables in $X$ such that the formula $\left.\forall Y G\right|_{v_{X}}$ is true. Here by $\left.G\right|_{v_{X}}$ we denote the formula obtained from $G$ by substituting the truth values given by $v_{X}$ for the corresponding variables from $X$, and then by simplifying these values away. We understand the formula $\left.F\right|_{v_{X}}$ in the same way. Clearly, $\left.\left.F\right|_{v_{X}} \equiv \neg G\right|_{v_{X}}$. Thus, $\left.\exists Y F\right|_{v_{X}}$ is false.

Let us define

$$
\begin{gathered}
E=\left\{\text { true }_{X}(a): a \in X \text { and } v_{X}(a)=\text { true }\right\} \cup \\
\{\text { choose }(a, 0): a \in Y\} .
\end{gathered}
$$

We will show that $(E, \emptyset)$ is a constrained explanation of goal. First, it is evident that $(E, \emptyset)$ is an explanation as goal can be derived through the first of its two rules. Next, we note that we cannot replace any constant $a$ appearing in atoms true $e_{X}(a)$ with a new constant $\xi$. Indeed, if true $(\xi)$ were to be a part of a the "add" part of an explanation, bad would hold (via the rule (13)) and goal would not! Similarly, we cannot replace $a \in Y$ in any atom choose $(a, 0)$, as only elements of $Y$ must show on these positions, the property forced by rule (12). Finally, we cannot replace any non-empty set of 0 's with $\xi$. If any such replacement resulted in an explanation, goal could only be derived through its second clause (allfalse cannot be derived now). However, that would imply that $\left.\exists Y F\right|_{v_{X}}$ is true, with the "witness" assignment assigning true to every $y \in Y$ such that $\operatorname{choose}(y, \xi)$ is a part of the modified explanation, and false to all other elements of $Y$.

$(\Leftarrow)$ Let us assume that goal has a constrained explanation. It must have a form $(E, \emptyset)$, where

$$
E=\left\{\operatorname{true}_{X}(a): a \in U\right\} \cup\left\{\operatorname{choose}(a, b): a \in Y, b \in U_{a}\right\},
$$

where $U$ is some subset of $X$ and where for every $a \in Y, U_{a}$, is some nonempty set. Indeed, if for some $a \in Y$, there is no $b$ such that $\operatorname{choose}(a, b) \in E, \operatorname{good}(a)$ cannot be derived from the revised program and, consequently, bad would follow (by the rule (15)). That would make it impossible to derive goal.

Now, if for some $a \in Y$ there is $b \in U_{a}$ such that $b \neq 0$, then $(E, \emptyset)$ is not constrained (indeed, that constant $b$ could be replaced by a new constant $\xi$ without any effect on the derivability of goal). Thus, for every $a \in Y, U_{a}=\{0\}$ and so,

$$
E=\left\{\text { true }_{X}(a): a \in U\right\} \cup\{\operatorname{choose}(a, 0): a \in Y\} .
$$


Since this explanation is constrained, there is no subset of positions where 0 occurs that can be substituted with $\xi$. Therefore, $\left.\exists Y F\right|_{v_{X}}$, where $v_{X}$ is the truth assignment determined by $U$, is false. One can show that by following the argument used in part (1) of the theorem (due to our assumption on $G$, the all-false assignment to atoms in $Y$ is not a model of $\left.F\right|_{v_{X}}$ ). Thus, $\Phi$ is true.

\section{Theorem 5}

Let $\mathscr{A}$ be a set of abducible predicates and $R$ a (fixed) Horn program with no abducible predicates in the heads of its rules.

1. The following problem is coNP-complete: given a set $B$ of abducibles, an observation $O$, and a pair $(E, F)$ of sets of abducibles, decide whether $(E, F)$ is a constrained explanation for $O$ wrt $\langle R \cup B, \mathscr{A}, \emptyset\rangle$.

2. The following problem is $\Sigma_{2}^{P}$-complete: given a set $B$ of abducibles and an observation $O$, decide whether a constrained explanation for $O$ wrt $\langle R \cup B, \mathscr{A}, \emptyset\rangle$ exists.

\section{Proof}

(1) The membership part follows by Theorem 3. To prove hardness, we show that the problem to decide whether $(E, F)$ is arbitrary is NP-hard. That is sufficient, as every explanation is either arbitrary or constrained. To show NP-hardness of the problem to decide whether an explanation is arbitrary, we reduce the SAT problem to it.

Thus, let $Y$ be a (finite) set of atoms, say $Y=\left\{y_{1}, \ldots, y_{n}\right\}$, and $F$ a CNF consisting of clauses $c_{1}, \ldots, c_{m}$. We denote by $C l(F)$ the set of clauses in $F$, that is, $C l(F)=\left\{c_{1}, \ldots, c_{m}\right\}$. Let us also consider three additional distinct symbols $t, f$ and 0 . We define the vocabulary $\sigma$ to consist of predicate symbols $i_{Y} / 1$, clause/1, pos/2, ngtd/2, p/2 true/1, false/0, ok/1, next/2, next f $_{2}$, clsat/0, sat/1, and goal/0, and an abductive theory

$$
\mathscr{T}(F)=\langle T(F),\{p\}, \emptyset\rangle,
$$

where $T(F)$ consists of the following atoms:

1. $\operatorname{in}_{Y}(a)$, for every $a \in Y \cup\{t, f\}$

2. $\operatorname{pos}(a, c)$, for every atom $a \in Y$ and clause $c \in C l(F)$ such that $a$ occurs non-negated in $c$

3. $n \operatorname{gtd}(a, c)$, for every atom $a \in Y$ and clause $c \in C l(F)$ such that $a$ occurs negated in $c$

4. $n \operatorname{ext}\left(y_{i}, y_{i+1}\right)$, for $i=1, \ldots, n-1, n \operatorname{ext}\left(t, y_{1}\right)$, and $\operatorname{next}\left(y_{n}, f\right)$

5. $\operatorname{next}_{C}\left(c_{i}, c_{i+1}\right)$, for $i=1, \ldots, m-1$

6. $p(t, 0)$

and of the following rules

1. $\operatorname{clause}(C) \leftarrow \operatorname{pos}(A, C)$

2. $\operatorname{clause}(C) \leftarrow \operatorname{ngtd}(A, C)$

3. $\operatorname{true}(A) \leftarrow \operatorname{in}_{Y}(A), p(A, Z), p(t, Z)$

4. false $(A) \leftarrow \operatorname{in}_{Y}(A), p(A, Z), p(f, Z)$

5. $\operatorname{clsat}(C) \leftarrow \operatorname{pos}(A, C), \operatorname{true}(A)$

6. $\operatorname{clsat}(C) \leftarrow \operatorname{ngtd}(A, C)$, false $(A)$

7. $o k(t)$

8. $\operatorname{ok}(A) \leftarrow \operatorname{ok}\left(A^{\prime}\right), \operatorname{next}\left(A^{\prime}, A\right)$, true $(A)$

9. $\operatorname{ok}(A) \leftarrow$ ok $\left(A^{\prime}\right), \operatorname{next}\left(A^{\prime}, A\right)$,false $(A)$

10. $o k(f) \leftarrow o k\left(A^{\prime}\right), \operatorname{next}\left(A^{\prime}, f\right)$ 


$$
\begin{aligned}
& \text { 11. } \operatorname{sat}\left(c_{1}\right) \leftarrow \operatorname{clsat}\left(c_{1}\right) \\
& \text { 12. } \operatorname{sat}(C) \leftarrow \operatorname{sat}\left(C^{\prime}\right), \operatorname{next}_{C}\left(C^{\prime}, C\right), \operatorname{clsat}(C) \\
& \text { 13. } \operatorname{goal} \leftarrow \operatorname{ok}(f), \operatorname{sat}\left(c_{m}\right), p(f, Z) .
\end{aligned}
$$

Clearly, the pair $(E, \emptyset)$, where $E=\{p(x, 0): x \in Y \cup\{f\}\}$, is an explanation of goal. Indeed, for every $x \in Y$, both true $(x)$ and false $(x)$ can be derived from $T(F) \cup E$ (because $p(t, 0)$ and $p(f, 0)$ both hold in $T(F) \cup E)$. Thus, for every clause $c, \operatorname{clsat}(C)$ can be derived, too. These two observations imply that $o k(f)$ and $\operatorname{sat}\left(c_{m}\right)$ can both be derived from $T(F) \cup E$. Consequently, goal is explained by $(E, \emptyset)$.

Let us assume that $E$ is arbitrary. We will prove that $F$ is satisfiable. By the definition, one of the constants appearing in $E$ can be replaced by a fresh constant $\xi$ so that the resulting pair $\left(E^{\prime}, \emptyset\right)$ is an explanation of goal wrt $\mathscr{T}(F)$. It follows that $o k(f)$ can be derived from $T(F) \cup E^{\prime}$, that is, that for every $x \in Y$, at least one of true $(x)$ and false $(x)$ can be derived. This, implies that for every $x \in Y, p(x, 0) \in T(F) \cup E^{\prime}$, that is, $\xi$ is substituted for $f$ or 0 in $E$.

Since, by (13), every explanation of goal contains at least one atom of the form $p(f, z), \xi$ is not substituted for $f$ in $E$ to produce $E^{\prime}$. Thus, $E^{\prime}$ is obtained from $E$ by substituting $\xi$ for some occurrences of 0 . Let $U=\left\{u \in Y \cup\{f\}: p(u, \xi) \in E^{\prime}\right\}$. If $f \notin U$, then let $y$ denote any element in $U \cap Y$ (such an element exists as $U \neq \emptyset$ ). Since $p(t, \xi)$ and $p(f, \xi)$ are not in $T(F) \cup E^{\prime}$, neither true $(y)$ nor false $(y)$ can be derived from $T(F) \cup E^{\prime}$. Thus, neither $o k(f)$ nor goal can be derived from $T(F) \cup E^{\prime}$. It follows that $f \in U$. Consequently, for every $x \in U$, false $(x)$ can be derived from $T(F) \cup E^{\prime}$, and true $(x)$ cannot be. Similarly, for every $x \in Y \backslash U$, true $(x)$ can be derived from $T(F) \cup E^{\prime}$, and false $(x)$ cannot be. Thus, the atoms true $(x)$ and false $(x)$ in $T(F) \cup E^{\prime}$ determine a truth assignment on atoms of $Y$. Since sat $\left(c_{m}\right)$ can be derived from $T(F) \cup E^{\prime}, \operatorname{clsat}(c)$ can be derived form $T(F) \cup E^{\prime}$, for every clause $c$ in $F$. It follows that the truth assignment determined by the atoms true $(x)$ and false $(x)$ in $T(F) \cup E^{\prime}$ satisfies $F$.

Conversely, let us assume that $F$ is satisfiable. Let us consider any satisfying assignment for $F$ and let $U$ comprises $f$ and those atoms in $Y$ that are false under this assignment. Let $E^{\prime}$ be obtained from $E$ by substituting $\xi$ for the occurrences of 0 in atoms $p(y, 0), y \in U$. It is easy to verify that for every $y \in U$, false $(y)$ can be derived from $T(F) \cup E^{\prime}$, and true $(y)$ cannot be. Similarly, for every $y \in Y \backslash U$, $\operatorname{true}(y)$ can be derived from $T(F) \cup E^{\prime}$, and false $(y)$ cannot be. Moreover, $\operatorname{clsat}(c)$ can be derived from $T(F) \cup E^{\prime}$, for every clause $c$ of $F$. Consequently, $o k(f)$ and $\operatorname{sat}\left(c_{m}\right)$ can be derived from $T(F) \cup E^{\prime}$. Since $p(f, \xi) \in E^{\prime}$, goal can be derived from $T(F) \cup E^{\prime}$, that is, $\left(E^{\prime}, \emptyset\right)$ is an explanation of goal wrt $\mathscr{T}$. Thus, $(E, \emptyset)$ is arbitrary.

(2) The argument for the membership part follows by Theorem 3

We prove hardness. The problem to decide whether a QBF $\Phi=\exists X \forall Y G$, where $G$ is a DNF formula over variables in $X \cup Y$, is true, is $\Sigma_{2}^{P}$-complete. We will reduce it to the problem in question.

Below, we understand $v_{X}, F, C l(F)$ and $\left.G\right|_{v_{X}}$ as in the proof of Theorem 4 We assume that $X=\left\{x_{1}, \ldots, x_{k}\right\}, Y=\left\{y_{1}, \ldots, y_{n}\right\}$ and $C=\left\{c_{1}, \ldots, c_{m}\right\}$.

We define $\sigma$ to consist of predicate symbols $\operatorname{in}_{X} / 1, i n_{Y} / 1$, clause $/ 1, p o s / 2, n g t d / 2$, next $_{X} / 2$, next $_{Y} / 2$, next $_{C} / 2$, true $_{X} / 1$, false $_{X} / 1$, true $/ 1$, false $/ 1$, ok $_{X} / 1$, ok $_{Y} / 1$, sat $/ 1, f_{X} / 1, l_{X} / 1, f_{Y} / 1$, $l_{Y} / 1, f_{C} / 1, l_{C} / 1, \operatorname{tr} / 1, f a / 1, \operatorname{assign} / 2, \operatorname{good}_{X} / 0, \operatorname{good}_{Y} / 0, \operatorname{good}_{C} / 0, \operatorname{good}_{f} / 0, \operatorname{goal} / 0$. We assume three new distinct constants $0, t$ and $f$ and consider an abductive theory

$$
\mathscr{T}(F)=\left\langle T(F),\left\{\text { true }_{X}, \text { false }_{X}, \text { assign }, f a\right\}, \emptyset\right\rangle,
$$

where $T(F)$ consists of the following atoms (part $B$ ): 
1. $\operatorname{in}_{X}(a)$, for every $a \in X$

2. $\operatorname{in}_{Y}(a)$, for every $a \in Y \cup\{t, f\}$

3. $\operatorname{pos}(a, c)$, for every atom $a \in X \cup Y$ and clause $c \in C l(F)$ such that $a$ occurs non-negated in $c$

4. $n \operatorname{gtd}(a, c)$, for every atom $a \in X \cup Y$ and clause $c \in C l(F)$ such that $a$ occurs negated in $c$

5. $f_{X}\left(x_{1}\right), l_{X}\left(x_{k}\right)$

6. $f_{Y}\left(y_{1}\right), l_{Y}\left(y_{n}\right)$

7. $f_{C}\left(c_{1}\right), l_{C}\left(c_{m}\right)$

8. $\operatorname{next}_{X}\left(x_{i}, x_{i+1}\right)$, for $i=1, \ldots, k-1$

9. $\operatorname{next}_{Y}\left(y_{i}, y_{i+1}\right)$, for $i=1, \ldots, n-1$

10. $\operatorname{next}_{C}\left(c_{i}, c_{i+1}\right)$, for $i=1, \ldots, m-1$

11. $\operatorname{tr}(0)$

and of the following rules (part $R$ )

1. $\operatorname{clause}(C) \leftarrow \operatorname{pos}(A, C)$

2. $\operatorname{clause}(C) \leftarrow \operatorname{ngtd}(A, C)$

3. $\operatorname{true}(A) \leftarrow$ true $_{X}(A)$

4. false $(A) \leftarrow$ false $_{X}(A)$

5. $\operatorname{true}(B) \leftarrow \operatorname{in}_{X}(B)$, true $_{X}(A)$, false $_{X}(A)$

6. false $(B) \leftarrow \operatorname{in}_{X}(B)$,rue $_{X}(A)$, false fr $_{X}(A)$

7. $\operatorname{true}(B) \leftarrow \operatorname{in}_{Y}(B)$, true $_{X}(A)$, false $_{X}(A)$

8. false $(B) \leftarrow \operatorname{in}_{Y}(B)$, true $_{X}(A)$, false ${ }_{X}(A)$

9. $\operatorname{true}(A) \leftarrow \operatorname{in}_{Y}(A), \operatorname{assign}(A, Z), \operatorname{tr}(Z)$

10. false $(A) \leftarrow \operatorname{in}_{Y}(A), \operatorname{assign}(A, Z), f a(Z)$

11. $\operatorname{clsat}(C) \leftarrow \operatorname{pos}(A, C), \operatorname{true}(A)$

12. $\operatorname{clsat}(C) \leftarrow \operatorname{ngtd}(A, C)$, false $(A)$

13. $\operatorname{ok}_{X}(A) \leftarrow f_{X}(A)$,true $(A)$

14. $\operatorname{ok}_{X}(A) \leftarrow f_{X}(A)$,false $(A)$

15. $\operatorname{ok}_{X}(A) \leftarrow o k_{X}\left(A^{\prime}\right), \operatorname{next}_{X}\left(A^{\prime}, A\right)$, true $(A)$

16. $\operatorname{ok}_{X}(A) \leftarrow o k_{X}\left(A^{\prime}\right), \operatorname{next}_{X}\left(A^{\prime}, A\right)$,false $(A)$

17. $\operatorname{good}_{X} \leftarrow \operatorname{ok}_{X}(A), l_{X}(A)$

18. $\operatorname{ok}_{Y}(A) \leftarrow f_{Y}(A)$, true $(A)$

19. $\operatorname{ok}_{Y}(A) \leftarrow f_{Y}(A)$,false $(A)$

20. $\operatorname{ok}_{Y}(A) \leftarrow \operatorname{ok}_{Y}\left(A^{\prime}\right), \operatorname{next}_{Y}\left(A^{\prime}, A\right)$, true $(A)$

21. $\operatorname{ok}_{Y}(A) \leftarrow \operatorname{ok}_{Y}\left(A^{\prime}\right), \operatorname{next}_{Y}\left(A^{\prime}, A\right)$,false $(A)$

22. $\operatorname{good}_{Y} \leftarrow \operatorname{ok}_{Y}(A), l_{Y}(A)$

23. $\operatorname{sat}(C) \leftarrow \operatorname{clsat}(C), f_{C}(C)$

24. $\operatorname{sat}(C) \leftarrow \operatorname{sat}\left(C^{\prime}\right), \operatorname{next}_{C}\left(C^{\prime}, C\right), \operatorname{clsat}(C)$

25. $\operatorname{good}_{C} \leftarrow \operatorname{sat}(C), l_{C}(C)$

26. $\operatorname{goal} \leftarrow \operatorname{good}_{X}, \operatorname{good}_{Y}, \operatorname{good}_{C}, f a(Z)$

27. $\operatorname{goal} \leftarrow \operatorname{good}_{X}, \operatorname{good}_{Y}, \operatorname{in}_{Y}(A)$,false $(A)$, true $(A)$, fa $(Z)$.

Let $v_{X}$ be an assignment of truth values to variables in $X$ such that the formula $\left.\forall Y G\right|_{v_{X}}$ is true, and let

$$
\begin{aligned}
E= & \left\{\text { true }_{X}(a): a \in X \text { and } v_{X}(a)=\text { true }\right\} \cup \\
& \left\{\text { false }_{X}(a): a \in X \text { and } v_{X}(a)=\text { false }\right\} \cup \\
& \{\text { assign }(y, 0): y \in Y\} \cup\{f a(0)\} .
\end{aligned}
$$


It is clear that $(E, \emptyset)$ is an explanation for goal wrt $\mathscr{T}(F)$. Indeed, since for every $y \in Y$ we have true $(y)$ and false (y), goal can be derived by means of the rule (27). Let us assume that $E$ is arbitrary. Then, there is a constant, say $a$, appearing in $E$ such that replacing some occurrences of $a$ with a fresh constant $\xi$ results in another explanation of goal. However, if $a \in X$, then neither true (a) nor false (a) can be derived after the replacement. Consequently, we cannot derive $\operatorname{good}_{X}$ and so, we cannot derive goal either. If $a \in Y$, then again neither true (a) nor false(a) can be derived. Now, $\operatorname{good}_{Y}$ cannot be derived and so, neither can goal. Thus, $a=0$. If we do not replace the occurrence of 0 in $f a(0)$ with $\xi$, then there is $y \in Y$ such that we replace the occurrence of 0 in $\operatorname{assign}(y, 0)$ with $\xi$. For that $y$, after the replacement we cannot derive true $(y)$ nor false $(y)$ and so, $\operatorname{good}_{Y}$ and goal cannot be derived. It follows that there is a set $Y^{\prime} \subseteq Y$ such that

$$
\begin{aligned}
E^{\prime}= & \left\{\text { true }_{X}(a): a \in X \text { and } v_{X}(a)=\text { true }\right\} \cup \\
& \left\{\text { false }_{X}(a): a \in X \text { and } v_{X}(a)=\text { false }\right\} \cup \\
& \left\{\text { assign }(y, 0): y \in Y \backslash Y^{\prime}\right\} \cup\left\{\operatorname{assign}(y, \xi): y \in Y^{\prime}\right\} \cup\{f a(\xi)\}
\end{aligned}
$$

gives rise to an explanation $\left(E^{\prime}, \emptyset\right)$ of goal. Clearly, after applying $\left(E^{\prime}, \emptyset\right)$, for no $y \in Y$, both true $(y)$ and false $(y)$ can be derived. Thus, goal must be derivable by means of the rule (26). Moreover, for every $y \in Y$, we have exactly one of true $(y)$ and false $(y)$ hold: $\operatorname{true}(y)$ holds in $y \in Y \backslash Y^{\prime}$, and false $(y)$ holds if $y \in Y^{\prime}$. Since goal can be derived, it follows that $\operatorname{good}_{C}$ can be derived. Consequently, the truth assignment on $Y$ defined by the atoms true $(y)$ and false $(y)$, where $y \in Y$, satisfies the set of clauses of $\left.F\right|_{v_{X}}$, that is $\left.\exists Y F\right|_{v_{X}}$ is true. This is a contradiction since $\left.\left.\exists Y F\right|_{v_{X}} \equiv \neg \forall Y G\right|_{v_{X}}$. Hence, $(E, \emptyset)$ is constrained.

Conversely, let $(E, \emptyset)$ be a constrained explanation of the goal. Clearly, $E$ consists of facts of the form $\operatorname{true}_{X}(a)$ false $_{X}(b)$, assign $(y, z)$ and $f a(w)$. For every element $x \in X$, at least one of $\operatorname{true}_{X}(x)$ and false $_{X}(x)$ must be present in $E$ (otherwise, we cannot derive $\operatorname{good}_{X}$ ). Moreover, if for at least one element $a \in X$ we have $\operatorname{true}_{X}(a)$ and false $_{X}(a)$ in $E$, then changing these two occurrences of $a$ to $\xi$ does not affect derivability of goal (indeed, by the rules (5)-(8) both before and after the change we have true $(x)$ and false $(x)$ hold for all $x \in X \cup Y)$. Thus, $(E, \emptyset)$ would not be constrained. Finally if true $_{X}(a)$ or false $e_{X}(a)$ is in $E, a \in X$. Otherwise, that $a$ could be replaced by $\xi$ without affecting the derivability of goal, contradicting again the assumption that $(E, \emptyset)$ is constraied. It follows that if $\operatorname{true}_{X}(x)$ or $\operatorname{false}_{X}(x)$ is in $E, x \in X$ and that the atoms $\operatorname{true}_{X}(x)$ and false $_{X}(x)$ that belong to $E$ determine a truth assignment on $X$, say $v_{X}$.

Next, let us assume that for some $\alpha \neq 0$ we have $\operatorname{assign}(y, \alpha) \in E$. Then replacing all occurrences of $\alpha$ by $\xi$ (including possibly an occurrence if $\alpha$ in $f a(\alpha)$ ) has no effect on the derivability of goal. As before, we get a contradiction. Thus, if $E$ contains facts assign $(y, z)$, they are of the form $\operatorname{assign}(y, 0)$. If any of these $y$ 's is not in $Y$, it can be changed to $\xi$ without affecting the derivability of goal.

Next, we note that if $E$ contains $f a(\alpha)$, where $\alpha \neq 0$, that $\alpha$ can be changed to $\xi$ without affecting the derivability of goal.

If for some $y \in Y, \operatorname{assign}(y, 0)$ is not in $E$, then for that $y$ we can derive neither true $(y)$ nor false $(y)$. Thus, we cannot derive $\operatorname{good}_{Y}$ and, consequently, we cannot derive goal either. It follows that $E$ contains all facts $\operatorname{assign}(y, 0), y \in Y$, and no other facts based on the relation symbol assign.

If $f a(0)$ is not in $E$, goal cannot be derived. Thus, $E$ is of the form we considered above. Let $Y^{\prime} \subseteq Y$ and let $E^{\prime}$ be as above. Since $(E, \emptyset)$ is constrained, $\left(E^{\prime}, \emptyset\right)$ is not an explanation of goal. That is a truth assignment on $Y$ such that elements in $Y \backslash Y^{\prime}$ are assigned true and those in $Y^{\prime}$ 
are assigned false is not a satisfying assingment for $\left.F\right|_{\nu_{X}}$. Consequently, it follows that $\left.\exists Y F\right|_{v_{X}}$ is false and so, $\left.\forall Y G\right|_{v_{X}}$ is true. This last property implies that $\exists X \forall Y G$ is true.

\section{Theorem 6}

Let $\mathscr{A}$ be a set of abducible predicates, $R$ a (fixed) non-recursive Horn program with no abducible predicates in the heads of its rules, and $\mathscr{C}$ a (fixed) set of integrity constraints.

1. The following problem is coNP-complete: given a set $B$ of abducibles, an observation $O$, and a pair $(E, F)$ of sets of abducibles, decide whether $(E, F)$ is a constrained explanation for $O$ wrt $\langle R \cup B, \mathscr{A}, \mathscr{C}\rangle$.

2. The following problem is $\Sigma_{2}^{P}$-complete: given a set $B$ of abducibles and an observation $O$, decide whether a constrained explanation for $O$ wrt $\langle R \cup B, \mathscr{A}, \mathscr{C}\rangle$ exists.

\section{Proof}

(1) The membership part follows by Theorem 3. To prove hardness, we show that the problem to decide whether $(E, F)$ is arbitrary is NP-hard. That is sufficient, as every explanation is either arbitrary or constrained. To show NP-hardness of the problem to decide whether an explanation is arbitrary, we reduce the SAT problem to it. Thus, let $Y$ be a (finite) set of atoms and $F$ a CNF theory over $Y$. As before, we denote by $C l(F)$ the set of clauses in $F$. Let us also consider three additional distinct symbols $t, f$ and 0 . We define the vocabulary $\sigma$ to consist of predicate symbols

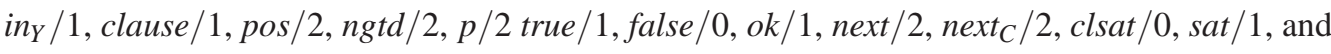
$\mathrm{goal} / 0$, and an abductive theory

$$
\mathscr{T}(F)=\langle T(F),\{p\}, \mathscr{C}\rangle
$$

where $T(F)$ consists of the following atoms:

1. $\operatorname{in}_{Y}(a)$, for every $a \in Y \cup\{t, f\}$

2. $\operatorname{pos}(a, c)$, for every atom $a \in Y$ and clause $c \in C l(F)$ such that $a$ occurs non-negated in $c$

3. $\operatorname{ngtd}(a, c)$, for every atom $a \in Y$ and clause $c \in C l(F)$ such that $a$ occurs negated in $c$

4. $p(t, 0)$

and of the rules

1. $\operatorname{clause}(C) \leftarrow \operatorname{pos}(A, C)$

2. $\operatorname{clause}(C) \leftarrow \operatorname{ngtd}(A, C)$

3. $\operatorname{true}(A) \leftarrow \operatorname{in}_{Y}(A), p(A, Z), p(t, Z)$

4. false $(A) \leftarrow \operatorname{in}_{Y}(A), p(A, Z), p(f, Z)$

5. $\operatorname{clsat}(C) \leftarrow \operatorname{pos}(A, C)$, true $(A)$

6. $\operatorname{clsat}(C) \leftarrow \operatorname{ngtd}(A, C)$, false $(A)$

7. goal $\leftarrow p(f, X)$

and where $\mathscr{C}$ consists of

1. $\forall C$ clause $(C) \supset \operatorname{clsat}(C)$

2. $\forall A \operatorname{in}_{Y}(A) \supset$ false $(A) \vee \operatorname{true}(A)$.

Clearly, the pair $(E, \emptyset)$, where $E=\{p(x, 0): x \in Y \cup\{f\}\}$, is an explanation of goal. Indeed, for every $x \in Y$, both true $(x)$ and false $(x)$ can be derived from $T(F) \cup E$ (because $p(t, 0)$ and $p(f, 0)$ both hold in $T(F) \cup E)$. Thus, for every clause $c, \operatorname{clsat}(C)$ can be derived, too. Consequently, the two integrity constraints in the theory hold for the least model of the program 
$T(F) \cup E$. Moreover, goal belongs to this unique model and so, it is entailed by the revised theory.

Let us assume that $E$ is arbitrary. We will prove that $F$ is satisfiable. By the definition of arbitrariness, one of the constants appearing in $E$ can be replaced by a fresh constant $\xi$ so that the resulting pair $\left(E^{\prime}, \emptyset\right)$ is an explanation of goal wrt $\mathscr{T}(F)$, that is, the least model of $T(F) \cup E^{\prime}$ satisfies both integrity constraints of the abductive theory and contains an atom of the form $p(f, z)$ (in order for goal to hold.

If $f$ is replaced with $\xi$ in $E$, then the least model of $T(F) \cup E^{\prime}$ does not contain any fact of the form $p(f, X)$, and $\left(E^{\prime}, \emptyset\right)$ is not an explanation. If some atom $x \in Y$ is replaced by $\xi$, then for that atom neither true $(x)$ nor false $(x)$ belongs to the least model of $T(F) \cup E^{\prime}$, which means that this model violates the second integrity constraint, contrary to the fact that $\left(E^{\prime}, \emptyset\right)$ is an explanation.

Thus, there is a non-empty set $U \subseteq Y \cup\{f\}$, such that when each occurrence of 0 in $p(x, 0)$, where $u \in U$, is replaced by $\xi$, the resulting set $E^{\prime}=\{p(x, \xi): x \in U\} \cup\{p(x, 0): x \in(Y \backslash U)\}$ gives rise to an explanation $\left(E^{\prime}, \emptyset\right)$. Let us assume that $f \notin U$. Since $U \neq \emptyset, U \cap Y \neq \emptyset$. Let $x \in U \cap Y$. For this $x$, the least model of $T(F) \cup E^{\prime}$ contains neither false $(x)$ nor true $(x)$, violating the second integrity constraint. Thus, $f \in U$ and, consequently, the least model of $T(F) \cup E^{\prime}$, contains atoms false $(x)$, where $x \in U \backslash\{f\}$, and true $(x)$, where $x \in Y \backslash U$. It follows that this set of atoms defines a valuation on $Y$. Moreover, since the first integrity constraint holds, this valuation satisfies all clauses of $F$.

Conversely, let us assume that $F$ is satisfiable. Let us consider any satisfying assignment for $F$ and let $U$ comprises $f$ and those atoms in $Y$ that are false under this assignment. Let $E^{\prime}$ be obtained from $E$ by substituting $\xi$ for the occurrences of 0 in atoms $p(y, 0), y \in U$. It is easy to verify that for every $y \in U$, false $(y)$ can be derived from $T(F) \cup E^{\prime}$, and true $(y)$ cannot be. Similarly, for every $y \in Y \backslash U$, true $(y)$ can be derived from $T(F) \cup E^{\prime}$, and false $(y)$ cannot be. Moreover, $\operatorname{clsat}(c)$ can be derived from $T(F) \cup E^{\prime}$, for every clause $c$ of $F$. Thus, both integrity constraints are satisfied by the least model of $T(F) \cup E^{\prime}$, and that model also contains $p(f, \xi)$ and so, also goal. Thus, $(E, \emptyset)$ is an arbitrary explanation of goal.

(2) The membership part follows by Theorem 3 . To prove hardness we proceed similarly as in the proofs of Theorems 4 and 5 .

\section{Theorem 7}

Let $\mathscr{A}$ be a set of abducible predicates and $R$ a (fixed) non-recursive Horn program with no abducible predicates in the heads of its rules. The following problems are in $\mathrm{P}$.

1. Given a set $B$ of abducibles, an observation $O$, and a pair $(E, F)$ of sets of abducibles, decide whether $(E, F)$ is a constrained explanation for $O$ wrt $\langle R \cup B, \mathscr{A}, \emptyset\rangle$.

2. Given a set $B$ of abducibles and an observation $O$, decide whether a constrained explanation for $O$ wrt $\langle R \cup B, \mathscr{A}, \emptyset\rangle$ exists.

\section{Proof}

(1) Let us consider an explanation $(E, F)$. Since $R$ is non-recursive, there is a constant, say $k$, such that any proof of $o$ based on the rules in $R$ and facts in $B$ revised by $(E, F)$ has length bounded from above by $k$. Thus, the total number of facts used in any such proof is bounded by $k$, too.

Since at most $k$ atoms in $E$ are relevant to any proof, if $E$ contains more than $k$ abducibles with predicate symbols of positive arity, it is not constrained. Indeed, at least one of these abducibles does not play any role in the proof. For for this abducible, say $a=p\left(c_{1}, \ldots, c_{m}\right)$, we have that replacing $c_{1}$ with $\xi$ in $a$ results in an explanation. 
If on the other hand, the number of abducibles with predicate symbols of positive arity in $E$ is less than or equal to $k$, then the total number of constants occurring in all abducibles in $E$ is bounded by a constant $k^{\prime}$ dependent on $R$ only (independent of the size of input, that is, of the size of $B$ ). Thus, there is only a fixed number of possible selections of occurrences of a constant for replacement by a new symbol $\xi$. For each of them, we can test in polynomial time whether it leads to an explanation. Thus, we can decide whether $(E, F)$ is constrained in polynomial time.

(2) If $(E, F)$ is a constrained explanation, then $(E, \emptyset)$ is a constrained explanation. Moreover, we can assume that $E$ contains all zero arity abducibles in $B$. Thus, each such constrained explanation is determined by its non-zero arity abducibles. Non-zero arity abducibles in a constrained explanation use only constants appearing in $O$ and $P$ (Theorem 11). It follows, the set of all possible non-zero arity abducibles that might be chosen to form $E$ has size that is polynomial in the size of $B$ (the input size). Since by an argument from the previous proof, we can assume that $E$ contains no more than $k$ non-zero arity abducibles (where $k$ is a constant depending only on $R$ ), the set of all candidate explanations that need to be tested to decide the problem is polynomial in the size of input. Since each such candidate explanation can be tested for constrainedness in polynomial time (by the previous result), the assertion follows. 